\title{
RADIAL VELOCITY CURVES OF ELLIPSOIDAL RED GIANT BINARIES IN THE LARGE MAGELLANIC CLOUD
}

\author{
J. D. NIE ${ }^{1,3}$ AND P. R. WoOD ${ }^{2}$ \\ ${ }^{1}$ Key Laboratory of Optical Astronomy, National Astronomical Observatories, Chinese Academy of Sciences, Beijing 100012, China; jdnie@ bao.ac.cn \\ ${ }^{2}$ Research School of Astronomy and Astrophysics, Australian National University, Cotter Road, Weston Creek, ACT 2611, Australia; peter.wood@anu.edu.au \\ Received 2014 May 21; accepted 2014 July 31; published 2014 November 4
}

\begin{abstract}
Ellipsoidal red giant binaries are close binary systems where an unseen, relatively close companion distorts the red giant, leading to light variations as the red giant moves around its orbit. These binaries are likely to be the immediate evolutionary precursors of close binary planetary nebula and post-asymptotic giant branch and post-red giant branch stars. Due to the MACHO and OGLE photometric monitoring projects, the light variability nature of these ellipsoidal variables has been well studied. However, due to the lack of radial velocity curves, the nature of their masses, separations, and other orbital details has so far remained largely unknown. In order to improve this situation, we have carried out spectral monitoring observations of a large sample of 80 ellipsoidal variables in the Large Magellanic Cloud and we have derived radial velocity curves. At least 12 radial velocity points with good quality were obtained for most of the ellipsoidal variables. The radial velocity data are provided with this paper. Combining the photometric and radial velocity data, we present some statistical results related to the binary properties of these ellipsoidal variables.
\end{abstract}

Key words: binaries: close - Magellanic Clouds - stars: AGB and post-AGB

Online-only material: color figures, figure set, machine-readable and VO tables

\section{INTRODUCTION}

The variable red giants in the Large Magellanic Cloud (LMC) fall on six or more distinct sequences in a period-luminosity (PL) diagram, i.e., $K-\log P$ diagram (Wood et al. 1999; Ita et al. 2004; Soszynski et al. 2007; Fraser et al. 2008). One of these sequences, sequence E, consists of binary systems that are mainly red giant ellipsoidal binaries (Wood et al. 1999; Soszyński et al. 2004). In these ellipsoidal binary systems, the red giant is the primary and it substantially fills its Roche lobe. The secondary, which is usually an unevolved mainsequence star, is unseen observationally in most cases. Due to the substantial filling of the Roche lobe, the red giant is distorted. Rotation of the distorted shape of the red giant as the binary progresses around its orbit causes a change in the apparent light seen by a distant observer. This leads to the characteristic light and velocity curves of ellipsoidal variables that have two cycles of light variation in one orbital period but only one cycle of radial velocity variation (e.g., Nicholls et al. 2010).

The sequence $\mathrm{E}$ stars are low-mass stars $\left(m<1.85 M_{\odot}\right)$ or intermediate-mass stars $\left(1.85 M_{\odot}<m<7.0 M_{\odot}\right)$. They can lie on either the red giant branch (RGB) or the asymptotic giant branch (AGB) and they are therefore in an evolutionary phase where the stellar radius is increasing. It is when the radius of the expanding red giant becomes a significant fraction of the binary separation that the ellipsoidal variability becomes detectable with current surveys such as MACHO and OGLE. The orbital periods of sequence $\mathrm{E}$ stars in the LMC lie in the range $\sim 30-1000$ days and the light amplitude is usually $<0.3 \mathrm{mag}$ in the MACHO red band $M_{R}$. Statistically, the sequence $\mathrm{E}$ stars make up approximately $0.5 \%-2 \%$ of the RGB and AGB stars in the LMC. About $7 \%$ of the sequence E stars are eclipsing and

\footnotetext{
3 J. D. Nie was a visitor at the Research School of Astronomy and Astrophysics of the Australian National University when this work was primarily done.
}

about $10 \%$ of them have unusually shaped light curves, which indicate significant eccentricity of the system orbits (Soszyński et al. 2004).

Due to the MACHO and OGLE projects, the variability nature of sequence E stars is well studied. However, at the present time, there are few radial velocity studies of the sequence $E$ stars, due to the difficulties of long-time spectral monitoring. Adams et al. (2006) observed two sequence E stars in the LMC, and their preliminary results indicated that the red giant is filling its Roche lobe and transferring mass to the companion since the derived mass of the red giant is close to that of the red giant core. Nicholls et al. (2010) carried out spectral observations on 11 sequence E stars (including the 2 in Adams et al. 2006) to derive radial velocity variations. The average full velocity amplitude derived for those 11 sequence $\mathrm{E}$ samples was $43.4 \mathrm{~km} \mathrm{~s}^{-1}$, consistent with the inference that sequence $\mathrm{E}$ stars are red giants in binary systems with roughly solar-mass components.

A problem with the current understanding of sequence $\mathrm{E}$ stars is that the eccentricity should be close to zero due to tidal interaction with the companion, yet about $10 \%$ of them have significant eccentricities (Soszyński et al. 2004). Complete orbital solutions for ellipsoidal red giant binaries with a range of eccentricities could possibly show in which part of the parameter space eccentricity can be maintained. As a start to such a study, Nicholls \& Wood (2012) monitored the radial velocities of seven eccentric sequence E stars and derived complete orbital solutions.

In general, a knowledge of the complete set of orbital properties (masses, separations, eccentricities, orientations) for a large sample of ellipsoidal red giant binaries in the LMC will provide a good resource for understanding the evolution of these close binary systems. These observed parameters can be used to constrain Monte Carlo simulations of the population of sequence E stars, such as those in Nie et al. (2012). These Monte Carlo calculations provide estimates for the production rate of binary post-AGB stars, binary planetary nebulae (PNe), 
binary post-RGB stars, and luminous white dwarfs relative to the production rate of single-star post-AGB stars and PNe. The relative numbers of the various types of objects listed above, now quite well known in the LMC, can be used to parameterize the binary interaction process, especially common envelope evolution, and to calibrate models for the formation of cataclysmic variable stars, AM CVn systems, the progenitors of SNe Ia, etc. So knowledge of the parameters of a large sample of the precursor binaries should help us better understand the interaction processes and the evolutionary fates of close red giant binaries.

In this study, we present the results of radial velocity monitoring of a large sample of ellipsoidal variables in the LMC. In Section 2, the design of our observing project and the data reduction is described. In Section 3, we present observed properties of binary stars based on the radial velocity and light variation data. Some statistical analyses are also provided and compared with theoretical models.

\section{OBSERVATIONS AND DATA REDUCTION}

\subsection{Selection of Objects}

We initially selected 86 sequence $E$ candidates from those given in Soszyński et al. (2004). Only objects with $I<16.5 \mathrm{mag}$ were considered since good radial velocities could not be obtained in a reasonable time (20 minute exposure) for fainter objects. Also, the data quality of fainter stars is relatively poor in the MACHO and OGLE databases, so their light curves have large dispersions and this is not good for fitting orbital solution.

All the sequence E candidates in Soszyński et al. (2004) and our 86 ellipsoidal variable candidates are plotted in the $I-\log P$ and $W-\log P$ planes in Figure 1 , where $W=I-1.55(V-I)$ is a reddening-free Weisenheit index (Madore 1982). It should also be noted that $P$ is the orbital period, which is twice the period obtained by Fourier analysis of raw light curves since ellipsoidal variables have two maxima and two minima of the light curve per orbital period. The sequence $E$ variables are often plotted in PL diagrams using the semi-period.

Our aim was to select a sample of objects with as wide a set of parameters as possible. We therefore selected objects randomly throughout sequence E, as shown in Figure 1, subject to the constraint $I<16.5$. Note that the magnitude cut of 16.5 is about 2 mag below the RGB tip, so our sample includes low-mass sequence $\mathrm{E}$ stars, even down to the masses of globular cluster red giants. We also made no attempt to select highly eccentric stars so the sample should include a range of eccentricities (note that Nicholls \& Wood 2012, have already studied a sample of seven eccentric sequence $\mathrm{E}$ stars).

At a given orbital period, more massive binary systems will have larger separations so the red giant will need to get to a higher luminosity (radius) before the Roche lobe filling factor is large enough to produce detectable ellipsoidal light variations. The more massive binaries should therefore preferentially lie in the high-luminosity side of sequence $\mathrm{E}$ in Figure 1. The results of Nicholls \& Wood (2012), shown in Figure 1, indicate that the higher-mass stars do indeed lie on the high-luminosity side of sequence E. In order to be sure of selecting a good sample of intermediate-mass stars $\left(M>\sim 1.85 M_{\odot}\right)$, we preferentially included extra stars above the blue line in Figure 1. Our final sample consists of 86 stars.

With 86 ellipsoidal candidates, plus 11 sequence E stars whose radial velocities were obtained by Nicholls et al. (2010) (green crosses in Figure 1) and the 7 eccentric sequence E stars

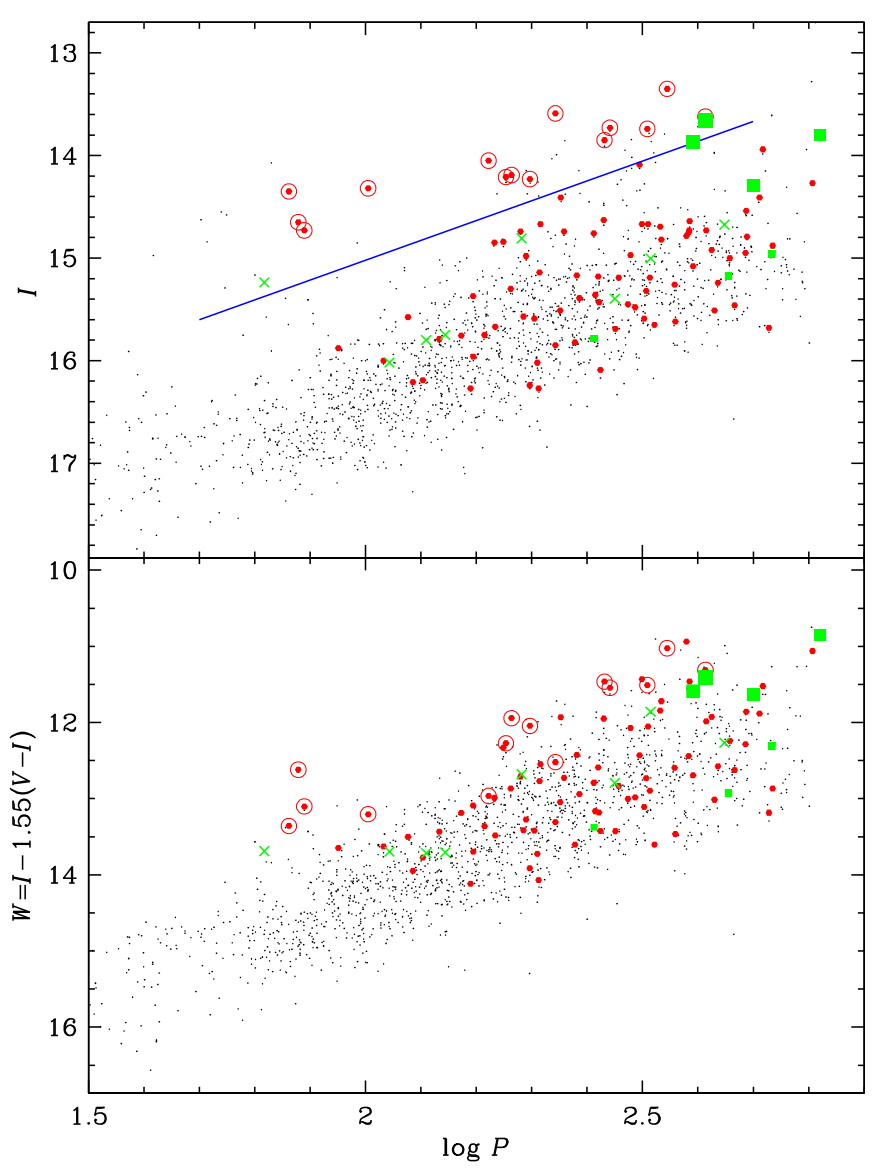

Figure 1. Location of our 86 ellipsoidal variable candidates (red dots) on the $I-\log P$ and $W-\log P$ planes. The small black dots show the full sample of ellipsoidal variables in Soszyński et al. (2004). The blue line in the top panel is a line parallel to the main part of sequence E. Stars above this line are brighter than normal for their period and are expected to be intermediate-mass stars. Objects in our sample that lie above the line are shown in both plots as circled points. The green squares are ellipsoidal variables studied by Nicholls \& Wood (2012) and the area of the square is proportional to the mass of the red giant derived by them. The green crosses are ellipsoidal variables studied by Nicholls et al. (2010). Due to the lack of $V$ band data, three objects of Nicholls et al. (2010) are absent.

(A color version of this figure is available in the online journal.)

studied by Nicholls \& Wood (2012) (green squares in Figure 1), we have a combined sample of approximately 100 . The full sample will give us statistical information about properties such as masses, mass ratio, eccentricity, separation, Roche lobe filling factor, and red giant luminosity for field red giants in the LMC. Derivation of some of these properties will require the use of a binary orbit modeling tool such as the Wilson-Devinney code (Wilson \& Devinney 1971; Wilson 1979, 1990; Wilson et al. 2009).

\subsection{Spectral Observation}

The radial velocity observations were taken using the Wide Field Spectrograph (WiFeS; Dopita et al. 2007, 2010) mounted on the Australian National University $2.3 \mathrm{~m}$ telescope at Siding Spring Observatory. WiFes is an integral field, double-beam, imaging-slicing spectrograph with a field of view $25 \times 38$ square arcsec, imaged onto 25 slits that are 1 arcsec wide and 38 arcsec long. It has six gratings, giving high $(R=7000)$ and low $(R=3000)$ spectral resolutions. For our observations, the gratings B7000 (wavelength coverage of 4184-5580 ) and I7000 (wavelength coverage of 6832-9120 ) were chosen for 
the blue and red CCD, respectively. These two gratings give a two-pixel resolution $R=7000$, corresponding to a $45 \mathrm{~km} \mathrm{~s}^{-1}$ velocity resolution.

We carried out 18 weeks of radial velocity monitoring, from 2010 September to 2012 March, to cover at least 1 orbital period for nearly all of the 86 objects. The observations were approximately evenly distributed throughout the 18 months, roughly 1 radial velocity observation per star per month. The exposure time was generally set to $300 \mathrm{~s}$ for objects with $I \sim 13 \mathrm{mag}, 600 \mathrm{~s}$ for $I \sim 14 \mathrm{mag}$, and $900 \mathrm{~s}$ for $I \sim 15 \mathrm{mag}$, which gives a signal-to-noise ratio $(\mathrm{S} / \mathrm{N})$ of at least 20 . For fainter objects $(I>16 \mathrm{mag})$, the exposure time was increased to $1200 \mathrm{~s}$. We also increased the exposure time when observing in bad weather, to guarantee a $\mathrm{S} / \mathrm{N}$ of 20.

For our observations, we chose the "stellar" mode exposure, in which case only 12 slits of the spectrograph were used. For flatfielding the QI-1 lamp was used and for wavelength calibration a $\mathrm{Ne}-\mathrm{Ar}$ arc lamp exposure was taken at the beginning of the night. For velocity derivation, the radial velocity standard star HR9014 was observed. In addition, the white dwarf star EG131 was observed so that telluric lines could be used to remove any zero point error in the wavelength calibration arising from spectrograph drift over the night. To achieve high $\mathrm{S} / \mathrm{N}$, the exposure times of HR9014 and EG131 were set to $10 \mathrm{~s}$ and $900 \mathrm{~s}$, respectively.

\subsection{Data Reduction}

\subsubsection{Spectrum Reduction}

The WiFes data reduction pipeline (Dopita et al. 2010) was used for the spectrum reduction. The pipeline combines the calibration and science data and provides one-dimensional spectra with most of the cosmic rays and sky emission lines removed. Since all our objects are red, most of their flux is concentrated in the red region of the spectrum. Therefore, we did not reduce the blue beam spectra because these spectra are of low S/N. The red spectra (6832-9120 $\AA$ ) contain many prominent telluric absorption lines as well as emission lines of water, $\mathrm{OH}$, and $\mathrm{O}_{2}$. The pipeline-reduced spectra sometimes contained some residual telluric lines and cosmic rays. Because of this, we checked by eye all the reduced spectra of the same object and, by comparison, removed residual cosmic rays and residual sky emission lines manually.

Spectrally, red giant binaries with white dwarf or neutron star companions always show characteristic emission lines (Allen 1984; Kenyon 1986; Mikolajewska et al. 1997; Belczyński et al. 2000). These objects are the symbiotic stars. In passing, we note that none of our ellipsoidal variables show emission lines in their spectra. This means that none of the companions are likely to be white dwarfs or neutron stars.

\subsubsection{Radial Velocity Calculation}

1. Relative radial velocity. The relative radial velocity and its error were computed using the IRAF fxcor package. The $f x$ cor package uses a Fourier cross-correlation method to find the wavelength shift between an object and a template spectrum in a specified cross-correlation region. The template was usually a radial velocity standard star with well-determined radial velocity and a spectral type similar to that of the program object. In our case, all the 86 ellipsoidal variables are stars of $\mathrm{K}-\mathrm{M}$ spectral type, so we choose as the template HR9014, which is a K5 star with a well-determined radial velocity of $-20.4 \mathrm{~km} \mathrm{~s}^{-1}$.
Table 1

Radial Velocities $v_{\mathrm{r}}$ and $1 \sigma$ Errors $\sigma_{v_{\mathrm{r}}}$ in $\mathrm{km} \mathrm{s}^{-1}$

\begin{tabular}{lccccccc}
\hline \hline \multicolumn{2}{c}{ OGLE 050659.79-692540.4 } & & \multicolumn{2}{c}{ OGLE 051256.36 -684937.5} \\
\cline { 1 - 2 } \cline { 6 - 7 } HJD(2450000+) & $v_{\mathrm{r}}$ & $\sigma_{v_{\mathrm{r}}}$ & & HJD $(2450000+)$ & $v_{\mathrm{r}}$ & $\sigma_{v_{\mathrm{r}}}$ \\
\hline 5463.06104 & 246.14 & 1.74 & & 5461.14209 & 237.26 & 1.70 \\
5513.99023 & 251.84 & 2.10 & & 5513.03125 & 247.21 & 1.64 \\
5563.00781 & 284.43 & 1.48 & & 5563.07324 & 257.77 & 1.54 \\
5590.98877 & 270.53 & 2.71 & & 5590.06592 & 256.17 & 2.27 \\
5603.96924 & 257.47 & 2.12 & & 5647.92578 & 243.94 & 1.51 \\
5649.00684 & 232.40 & 1.40 & & 5673.95508 & 229.63 & 2.68 \\
5678.97363 & 264.23 & 4.53 & & 5693.92676 & 230.36 & 1.53 \\
5679.01221 & 250.28 & 4.04 & & 5765.23242 & 231.89 & 2.03 \\
5764.26514 & 246.01 & 3.65 & & 5846.08301 & 242.70 & 1.92 \\
5783.19287 & 239.24 & 1.88 & & 5867.01807 & 252.29 & 1.39 \\
5848.15039 & 273.73 & 7.73 & & 5907.19336 & 257.03 & 1.75 \\
5868.03613 & 282.79 & 2.54 & & 5944.04883 & 255.03 & 1.35 \\
5906.11133 & 270.63 & 1.82 & & 5991.98584 & 243.43 & 1.50 \\
5944.17676 & 242.22 & 3.00 & & $\ldots$ & $\ldots$ & $\ldots$ \\
\hline
\end{tabular}

Note. Stars are identified by their OGLE II R.A. and decl.

(This table is available in its entirety in machine-readable and Virtual Observatory (VO) forms in the online journal. A portion is shown here for guidance regarding its form and content.)

The cross correlation was made on the wavelength interval 8400-8750 $\AA$ which contains the Ca II triplet lines and is relatively free of telluric lines. The heliocentric radial velocity $\left(v_{\text {helio }}(\mathrm{obj})\right)$, obtained from $f x$ cor after heliocentric correction and the inclusion of the heliocentric radial velocity of HR9014, was saved along with its error. The error in $v_{\text {helio }}(\mathrm{obj})$ is normally below $4 \mathrm{~km} \mathrm{~s}^{-1}$. If the error was larger than $10 \mathrm{~km} \mathrm{~s}^{-1}$ (due to bad weather and low $\mathrm{S} / \mathrm{N}$ ), then the velocity point was excluded from our data set.

2. Zero-point correction. To check the velocity calibration, all spectra of each program star were cross correlated with a template consisting of a single spectrum of the telluric standard star EG131. EG131 is a white dwarf that radiates almost like a blackbody. Thus, its spectrum has only telluric lines, making it an ideal template for the zeropoint correction. In principle, the relative velocity of the telluric lines in different spectra should be zero, but, due to the movement of the CCD system and spectrograph between the taking of the arc spectrum at the beginning of the night and the taking of the object spectrum, as well as the changes of the atmospheric pressure, there can be shifts in velocity. To compute this zero-point velocity shift $v_{\text {zp }}(\mathrm{obj})$, program stars were cross correlated with EG131 in the wavelength interval of $8120-8370 \AA$. This region is dominated by telluric lines and it is close to the region of Ca II triplet, so the zero-point correction in this region should similar to that of $\mathrm{Ca}$ II triplet region. The zero-point velocity correction varied from about -10 to $34 \mathrm{~km} \mathrm{~s}^{-1}$ across the many nights of the observation. HR9014 was also cross correlated with EG131 to obtain its zero point velocity correction $\left(v_{\text {zp }}(\right.$ HR9014)) .

3. Absolute radial velocity. To compute the absolute value for the observed radial velocity $v_{\mathrm{r}}$ including a zero-point correction, we use the formula

$$
v_{\mathrm{r}}=v_{\text {helio }}(\mathrm{obj})-v_{\mathrm{zp}}(\mathrm{obj})+v_{\mathrm{zp}}(\mathrm{HR} 9014) .
$$

The final radial velocity data for two objects are given in Table 1. The full table is available in the online journal. 
Table 2

Properties of the Observed Ellipsoidal Variables

\begin{tabular}{|c|c|c|c|c|c|c|c|c|c|c|c|c|c|}
\hline No. & $\begin{array}{c}\text { Object } \\
\text { (OGLE II Name) }\end{array}$ & $\begin{array}{c}P \\
\text { (day) }\end{array}$ & $\begin{array}{c}I \\
(\mathrm{mag})\end{array}$ & $\begin{array}{c}V \\
(\mathrm{mag})\end{array}$ & $\begin{array}{c}M_{B} \\
(\mathrm{mag})\end{array}$ & $\begin{array}{c}M_{R} \\
(\mathrm{mag})\end{array}$ & $\begin{array}{c}K \\
(\mathrm{mag})\end{array}$ & $\begin{array}{c}\Delta I \\
(\mathrm{mag})\end{array}$ & $\begin{array}{c}\Delta \mathrm{RV} \\
\left(\mathrm{km} \mathrm{s}^{-1}\right)\end{array}$ & $\begin{array}{c}L \\
\left(L_{\odot}\right)\end{array}$ & $\begin{array}{l}T_{\text {eff }} \\
(\mathrm{K})\end{array}$ & $\begin{array}{c}R \\
\left(R_{\odot}\right)\end{array}$ & Remark \\
\hline 1 & $050107.08-692036.9$ & 166.8 & 14.05 & 14.75 & $\ldots$ & $\cdots$ & 13.14 & 0.110 & 70 & 3278 & 5773 & 58 & 2,ogle 3 \\
\hline 2 & $050222.40-691733.6$ & 107.8 & 16.00 & 17.53 & 16.85 & 15.87 & 13.90 & 0.070 & 32 & 581 & 3990 & 51 & 1 \\
\hline 3 & $050254.15-692013.8$ & 171.5 & 15.67 & 17.08 & $\ldots$ & $\cdots$ & 13.82 & 0.025 & 35 & 736 & 4234 & 51 & 1 \\
\hline 4 & $050258.71-684406.6$ & 433.8 & 15.24 & 16.96 & 16.85 & 15.65 & 13.18 & 0.040 & 38 & 1149 & 4020 & 71 & 1 \\
\hline 5 & $050334.97-685920.5$ & 101.1 & 14.32 & 15.04 & 14.85 & 14.40 & 13.36 & 0.200 & 110 & 2526 & 5666 & 53 & 2 \\
\hline 6 & $050350.55-691430.2$ & 224.7 & 15.51 & 17.10 & 16.85 & 15.78 & 13.46 & 0.060 & 24 & 896 & 4030 & 62 & 1 \\
\hline 7 & $050353.41-690230.8$ & 323.6 & 14.67 & 16.36 & 16.28 & 15.13 & 12.65 & 0.060 & 7 & 1915 & 4049 & 90 & 1, ogle 3 \\
\hline 8 & $050438.97-693115.3$ & 383.2 & 14.75 & 16.24 & $\ldots$ & $\ldots$ & 12.99 & 0.070 & 14 & 1673 & 4321 & 74 & 1 \\
\hline 9 & $050454.49-690401.2$ & 220.2 & 13.59 & 14.28 & $\cdots$ & 13.72 & 12.79 & 0.070 & 120 & 5175 & 6018 & 67 & 2, ogle 3 \\
\hline 10 & $050504.70-683340.3$ & 238.9 & 15.82 & 17.25 & 16.95 & 16.00 & 14.05 & 0.040 & 40 & 631 & 4327 & 45 & 1 \\
\hline 11 & $050512.19-693543.5$ & 204.3 & 16.02 & 17.50 & 17.30 & 16.33 & 13.92 & 0.050 & 40 & 570 & 3988 & 51 & 1 \\
\hline 12 & $050554.57-683428.5$ & 270.1 & 13.85 & 15.39 & 15.10 & 14.07 & 11.85 & 0.035 & 26 & 4028 & 4061 & 129 & 2 \\
\hline 13 & $050558.70-682208.9$ & 264.0 & 15.43 & 16.88 & $\cdots$ & $\cdots$ & 13.48 & 0.020 & 22 & 938 & 4128 & 60 & 1 \\
\hline 14 & $050604.99-681654.9$ & 201.7 & 15.59 & 16.99 & 17.01 & 15.98 & 13.69 & 0.030 & 30 & 802 & 4174 & 55 & 1 \\
\hline 15 & $050610.03-683153.0$ & 318.5 & 15.59 & 17.19 & $\ldots$ & $\ldots$ & 13.71 & 0.030 & 35 & 798 & 4197 & 54 & $1, \mathrm{e}$ \\
\hline 16 & $050651.36-695245.4$ & 220.2 & 15.85 & 17.49 & 17.30 & 16.13 & 13.96 & 0.045 & 30 & 630 & 4192 & 48 & 1 \\
\hline 17 & $050659.79-692540.4$ & 156.4 & 15.37 & 16.84 & 16.74 & 15.75 & 13.54 & 0.070 & 50 & 963 & 4254 & 58 & 1 \\
\hline 18 & $050709.66-683824.8$ & 206.1 & 15.14 & 16.67 & 16.65 & 15.60 & 13.19 & 0.060 & 37 & 1223 & 4121 & 69 & 1 \\
\hline 19 & $050720.82-683355.2$ & 521.4 & 13.94 & 15.50 & 15.53 & 14.42 & 11.95 & 0.020 & 30 & 3699 & 4072 & 123 & 2 \\
\hline 20 & $050758.17-685856.3$ & 198.1 & 14.23 & 15.64 & 15.50 & 14.56 & 12.37 & 0.050 & 45 & 2744 & 4210 & 99 & 1 \\
\hline 21 & $050800.52-685800.8$ & 276.2 & 13.73 & 15.14 & 15.02 & 14.06 & 11.87 & 0.050 & 42 & 4337 & 4199 & 126 & 2 \\
\hline 22 & $050843.38-692815.1$ & 121.8 & 16.21 & 17.67 & 17.30 & 16.32 & 14.23 & 0.040 & 23 & 464 & 4098 & 43 & 1 \\
\hline 23 & $050900.02-690427.2$ & 156.5 & 15.96 & 17.42 & 17.25 & 16.23 & 14.14 & 0.025 & 16 & 561 & 4273 & 44 & 1 \\
\hline 24 & $050948.63-690157.4$ & 75.60 & 14.65 & 15.96 & 15.80 & 14.85 & 13.06 & 0.200 & 26 & 1779 & 4529 & 69 & 2 \\
\hline 25 & $051009.20-690020.0$ & 321.3 & 15.32 & 16.99 & 16.79 & 15.63 & 13.27 & 0.027 & 20 & 1067 & 4024 & 68 & $1, \mathrm{e}$ \\
\hline 26 & $051050.92-692228.0$ & 326.2 & 15.19 & 16.67 & 16.40 & 15.47 & 13.39 & 0.035 & 14 & 1128 & 4284 & 62 & 1, ogle 3 \\
\hline 27 & $051101.04-691425.1$ & 282.5 & 15.69 & 17.15 & 16.95 & 15.97 & 13.78 & 0.030 & 20 & 732 & 4173 & 52 & $1, \mathrm{e}$ \\
\hline 28 & $051200.23-690838.4$ & 641.3 & 14.27 & 16.34 & 16.13 & 14.80 & 11.89 & 0.030 & 14 & 3131 & 3737 & 135 & 1,e+sr,ogle 3 \\
\hline 29 & $051205.44-684559.9$ & 390.5 & 15.08 & 16.62 & 16.57 & 15.54 & 12.90 & 0.060 & 27 & 1382 & 3912 & 82 & 1,ogle3 \\
\hline 30 & $051220.63-684957.8$ & 486.5 & 14.54 & 16.27 & 16.10 & 14.92 & 12.45 & 0.070 & 19 & 2202 & 3983 & 99 & 1,e+sr,ogle 3 \\
\hline 31 & $051256.36-684937.5$ & 341.9 & 14.82 & 16.82 & 16.60 & 15.28 & 12.50 & 0.130 & 29 & 1851 & 3784 & 101 & 1, ogle 3 \\
\hline 32 & $051345.17-692212.1$ & 197.9 & 16.24 & 17.74 & 17.50 & 16.38 & 14.19 & 0.040 & 42 & 459 & 4038 & 44 & 1 \\
\hline 33 & $051347.73-693049.7$ & 306.6 & 15.48 & 17.09 & $\cdots$ & $\cdots$ & 13.52 & 0.040 & 20 & 899 & 4114 & 60 & $1, \mathrm{e}$ \\
\hline 34 & $051515.95-685958.1$ & 322.7 & 13.74 & 15.18 & 15.02 & 13.98 & 11.91 & 0.030 & 24 & 4269 & 4229 & 123 & $2, \mathrm{e}$ \\
\hline 35 & $051620.47-690755.3$ & 240.7 & 15.17 & 16.94 & 16.85 & 15.58 & 13.05 & 0.150 & 40 & 1249 & 3963 & 76 & 1, ogle 3 \\
\hline 36 & $051621.07-692929.6$ & 179.3 & 14.21 & 15.46 & 15.17 & 14.36 & 12.43 & 0.030 & 17 & 2748 & 4294 & 96 & 2 \\
\hline 37 & $051653.08-690651.2$ & 269.2 & 14.63 & 16.36 & 16.35 & 14.93 & 12.32 & 0.080 & 29 & 2193 & 3792 & 110 & 1 \\
\hline 38 & $051738.19-694848.4$ & 297.7 & 15.45 & 17.03 & 16.80 & 15.73 & 13.31 & 0.050 & 29 & 975 & 3943 & 68 & $1, \mathrm{e}$ \\
\hline 39 & $051746.55-691750.2$ & 225.2 & 14.41 & 16.01 & $\ldots$ & $\ldots$ & 12.33 & 0.050 & 15 & 2478 & 3985 & 105 & 1 \\
\hline 40 & $051818.84-690751.3$ & 463.9 & 15.46 & 17.29 & $\cdots$ & $\cdots$ & 13.03 & 0.050 & 26 & 1076 & 3710 & 80 & $1, \mathrm{e}$ \\
\hline 41 & $051845.02-691610.5$ & 320.0 & 15.65 & 16.97 & $\cdots$ & $\cdots$ & 13.90 & 0.040 & 55 & 734 & 4349 & 48 & 1 \\
\hline 42 & $052012.26-694417.5$ & 177.4 & 14.84 & 16.46 & $\cdots$ & 15.23 & 12.75 & 0.040 & 18 & 1675 & 3983 & 87 & 1 \\
\hline 43 & $052029.90-695934.1$ & 171.0 & 14.85 & 16.05 & 15.88 & 15.00 & 13.23 & 0.045 & 50 & 1489 & 4492 & 64 & 1 \\
\hline 44 & $052032.29-694224.2$ & 72.70 & 14.35 & 14.99 & 14.73 & 14.31 & 13.52 & 0.060 & 60 & 2569 & 5973 & 48 & $2, \mathrm{e}$ \\
\hline 45 & $052048.62-704423.5$ & 488.5 & 14.79 & $\cdots$ & $\cdots$ & $\cdots$ & 12.95 & 0.030 & 5 & 1641 & 4231 & 76 & 1 \\
\hline 46 & $052115.05-693155.1$ & 194.9 & 14.98 & 16.08 & 15.78 & 15.03 & 13.38 & 0.020 & 49 & 1318 & 4525 & 60 & 1 \\
\hline 47 & $052117.49-693124.8$ & 243.3 & 15.39 & 16.97 & 16.60 & 15.55 & 13.21 & 0.060 & 38 & 1044 & 3908 & 71 & 1 \\
\hline 48 & $052119.56-710022.1$ & 300.9 & 14.97 & 16.84 & 16.65 & 15.40 & 12.79 & 0.010 & 23 & 1531 & 3907 & 86 & 1, ogle 3 \\
\hline 49 & $052203.16-704507.8$ & 362.5 & 15.62 & 17.01 & 16.72 & 15.82 & 14.04 & 0.035 & 14 & 734 & 4564 & 44 & 1 \\
\hline 50 & $052228.85-694313.7$ & 231.0 & 14.74 & 16.04 & 15.77 & 14.85 & 12.99 & 0.030 & 37 & 1684 & 4334 & 74 & 1 \\
\hline 51 & $052238.43-691715.1$ & 77.53 & 14.73 & 15.78 & 15.41 & 14.74 & 13.47 & 0.070 & 55 & 1638 & 5046 & 53 & 2 \\
\hline 52 & $052324.57-692924.2$ & 312.4 & 14.09 & 15.16 & 15.08 & 14.43 & 12.39 & 0.030 & 40 & 3016 & 4388 & 96 & 2 \\
\hline 53 & $052422.28-692456.2$ & 485.4 & 14.95 & 16.67 & 16.65 & 15.49 & 12.82 & 0.060 & 18 & 1535 & 3948 & 85 & $1,+$ sr,ogle 3 \\
\hline 54 & $052425.52-695135.2$ & 206.7 & 14.67 & 16.04 & 15.87 & 15.03 & 12.93 & 0.040 & 42 & 1792 & 4345 & 75 & 1 \\
\hline 55 & 052438.19-700435.9 & 192.8 & 15.57 & 16.96 & 16.70 & 15.87 & 13.79 & 0.040 & 25 & 794 & 4314 & 51 & 1 \\
\hline 56 & $052438.40-700028.8$ & 410.8 & 13.62 & 15.11 & 14.83 & 14.00 & 11.71 & 0.060 & 50 & 4852 & 4147 & 136 & $2, \mathrm{e}$ \\
\hline 57 & $052458.88-695107.0$ & 340.6 & 14.67 & 16.53 & 16.33 & 15.20 & 12.35 & 0.075 & 5 & 2117 & 3790 & 108 & $1,+$ sr,ogle 3 \\
\hline 58 & $052510.82-700123.9$ & 189.7 & 14.74 & 16.05 & 15.87 & 15.05 & 12.99 & 0.030 & 30 & 1686 & 4328 & 74 & 1 \\
\hline 59 & $052513.34-693025.2$ & 259.4 & 14.76 & 16.03 & 15.97 & 15.14 & 13.12 & 0.030 & 45 & 1621 & 4471 & 68 & 1 \\
\hline 60 & $052542.11-694847.4$ & 514.6 & 14.41 & 16.04 & 15.90 & 14.85 & 12.35 & 0.030 & 35 & 2461 & 4004 & 104 & $1, \mathrm{e}$ \\
\hline 61 & $052554.91-694137.4$ & 182.8 & 15.30 & 16.87 & 16.95 & 15.85 & 13.23 & 0.120 & 40 & 1092 & 4008 & 69 & 1 \\
\hline 62 & $052703.65-694837.7$ & 350.4 & 13.35 & 14.85 & 14.70 & 13.78 & 11.43 & 0.100 & 60 & 6230 & 4131 & 156 & 2 \\
\hline 63 & $052833.50-695834.6$ & 183.2 & 14.19 & 15.64 & 15.65 & 14.75 & 12.31 & 0.060 & 20 & 2861 & 4186 & 103 & 2 \\
\hline 64 & $052928.90-701244.2$ & 531.5 & 15.68 & 17.29 & $\cdots$ & $\cdots$ & 13.71 & 0.060 & 25 & 751 & 4105 & 55 & $1, \mathrm{e}$ \\
\hline 65 & $052948.84-692318.7$ & 421.7 & 14.92 & 16.85 & 16.55 & 15.35 & 12.70 & 0.070 & 16 & 1625 & 3873 & 90 & 1,e+sr,ogle 3 \\
\hline
\end{tabular}


Table 2

(Continued)

\begin{tabular}{|c|c|c|c|c|c|c|c|c|c|c|c|c|c|}
\hline No. & $\begin{array}{c}\text { Object } \\
\text { (OGLE II Name) }\end{array}$ & $\begin{array}{c}P \\
\text { (day) }\end{array}$ & $\begin{array}{c}I \\
(\mathrm{mag})\end{array}$ & $\begin{array}{c}V \\
\text { (mag) }\end{array}$ & $\begin{array}{c}M_{B} \\
(\mathrm{mag})\end{array}$ & $\begin{array}{c}M_{R} \\
(\mathrm{mag})\end{array}$ & $\begin{array}{c}K \\
(\mathrm{mag})\end{array}$ & $\begin{array}{c}\Delta I \\
(\mathrm{mag})\end{array}$ & $\begin{array}{c}\Delta \mathrm{RV} \\
\left(\mathrm{km} \mathrm{s}^{-1}\right)\end{array}$ & $\begin{array}{c}L \\
\left(L_{\odot}\right)\end{array}$ & $\begin{array}{l}T_{\text {eff }} \\
(\mathrm{K})\end{array}$ & $\begin{array}{c}R \\
\left(R_{\odot}\right)\end{array}$ & Remark \\
\hline 66 & $052954.82-700622.5$ & 154.7 & 16.27 & 17.66 & 17.48 & 16.63 & 14.33 & 0.050 & 40 & 434 & 4149 & 41 & 1 \\
\hline 67 & $053141.27-700647.1$ & 384.1 & 14.73 & 16.78 & $\ldots$ & $\ldots$ & 12.36 & 0.090 & 24 & 2044 & 3751 & 108 & 1,e+sr,ogle 3 \\
\hline 68 & $053156.08-693123.0$ & 412.2 & 14.73 & 16.50 & $\cdots$ & $\cdots$ & 12.57 & 0.070 & 22 & 1899 & 3916 & 96 & 1,e+sr,ogle 3 \\
\hline 69 & $053202.44-693209.2$ & 426.5 & 15.51 & 17.12 & $\cdots$ & $\cdots$ & 13.48 & 0.030 & 25 & 892 & 4042 & 62 & $1, \mathrm{e}$ \\
\hline 70 & $053219.66-695805.0$ & 89.55 & 15.85 & 17.32 & 16.70 & 15.90 & 12.14 & 0.010 & 18 & 1749 & 3264 & 132 & 1 \\
\hline 71 & $053226.48-700604.7$ & 454.7 & 15.00 & 16.78 & 16.43 & 15.28 & 12.72 & 0.070 & 40 & 1547 & 3817 & 91 & 1,e+sr,ogle 3 \\
\hline 72 & $053337.07-703111.7$ & 315.5 & 14.67 & 16.76 & 16.60 & 15.28 & 12.37 & 0.130 & 16 & 2108 & 3799 & 107 & 1,e,ogle3 \\
\hline 73 & 053338.94-694455.2 & 126.9 & 16.19 & 17.75 & 17.60 & 16.51 & 14.23 & 0.090 & 32 & 469 & 4128 & 43 & 1 \\
\hline 74 & 053356.79-701919.6 & 205.3 & 16.27 & 17.69 & 17.53 & 16.55 & 14.35 & 0.040 & 40 & 432 & 4169 & 40 & 1 \\
\hline 75 & $053438.78-695634.1$ & 384.7 & 14.64 & 16.69 & 16.35 & 15.07 & 12.28 & 0.070 & 19 & 2218 & 3751 & 113 & 1, ogle 3 \\
\hline 76 & $053733.21-695026.9$ & 263.0 & 15.18 & 16.85 & 16.84 & 15.63 & 13.07 & 0.050 & 19 & 1235 & 3970 & 75 & 1 \\
\hline 77 & $053946.88-704257.8$ & 265.0 & 16.09 & 17.81 & 17.78 & 16.55 & 13.93 & 0.040 & 41 & 547 & 3929 & 51 & 1 \\
\hline 78 & $054006.47-702820.4$ & 361.5 & 15.26 & 16.98 & 16.85 & 15.63 & 13.08 & 0.070 & 29 & 1176 & 3906 & 76 & 1,e,ogle 3 \\
\hline 79 & $054258.34-701609.2$ & 164.0 & 15.75 & 17.29 & $\ldots$ & $\ldots$ & 13.79 & 0.040 & 20 & 703 & 4114 & 53 & 1 \\
\hline 80 & $054736.16-705627.2$ & 135.9 & 15.79 & 17.31 & 17.05 & 16.03 & 13.96 & 0.030 & 35 & 657 & 4253 & 48 & 1 \\
\hline
\end{tabular}

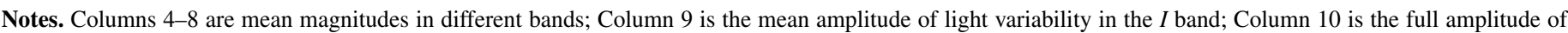

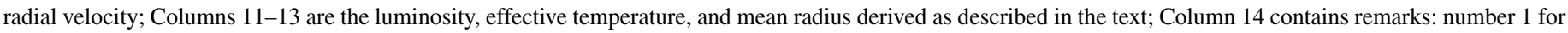

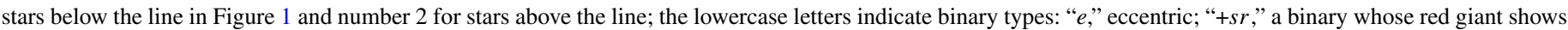
semi-regular variability as well as ellipsoidal variability; and "ogle3" denotes an object which has OGLE III light curve data.

(This table is also available in machine-readable and Virtual Observatory (VO) forms in the online journal.)

\section{RESULTS}

\subsection{Properties of the Observed Ellipsoidal Variables}

Before presenting the properties of the observed ellipsoidal variables, we need to remove candidates that are not clearly ellipsoidal variables with the help of the observed radial velocity. Among our 86 objects, we found that 80 of them are real ellipsoidal variables, showing two light maxima and two minima, but only one velocity maximum and minimum in one orbital period. The remaining six candidates do not clearly satisfy this requirement due to their low-velocity amplitude relative to the noise so they were removed. These six objects could be ellipsoidal variables whose orbital plane lies close to the plane of the sky or they could be sequence D stars as these stars lie close to the sequence $\mathrm{E}$ stars in the $I-\log P$ and $W-\log P$ planes and they have small velocity amplitudes (Nicholls et al. 2009). For the remainder of this paper, we focus on the 80 real ellipsoidal variables while we discuss the six rejected objects in the Appendix.

Table 2 shows various properties of the 80 ellipsoidal variables. For all objects, the period given has been re-derived because the original value from Soszyński et al. (2004) is not accurate enough. The MACHO and OGLE II light curve data were taken more than 10 years before our radial velocity data, which was obtained between years 2010 and 2012. Because of the long time span between the two sets of data, a small error in the period can cause a significant error in the phase of the light curve projected forward by more than 10 years. To obtain a more reliable period, when OGLE III data existed, we combined OGLE III and OGLE II light curves (covering the interval 1998-2009) and used the phase dispersion minimization method (Stellingwerf 1978) to calculate the period from the combined light curve. If the OGLE III data was not available for an object, we combined the OGLE II and MACHO data, covering the interval 1992-2000.

The effective temperature was calculated by converting $(I-K)_{0}$ to $T_{\text {eff }}$ using spline fits to the data in Houdashelt et al. (2000a, 2000b). The reason we used $(I-K)_{0}$ as the color index is because most of our sequence E stars are K- or early-M-type stars, and for these red giants $I-K$ varies much more with $T_{\text {eff }}$ than $J-K$ so that photometric errors are less important for $I-K$. The mean $I$ magnitude was derived from the OGLE photometry, while the $K$ magnitude was obtained from the Two Micron All Sky Survey catalog (Cutri et al. 2003). To remove reddening, we adopted $E(B-V)=0.08$ (Keller \& Wood 2006) along with $E(V-I)=1.38 \times E(B-V)$ (Schlegel et al. 1998), and $E(V-K)=2.744 \times E(B-V)$ and $A(K)=0.35 \times E(B-V)$ (Rieke \& Lebofsky 1985). The bolometric correction $\mathrm{BC}_{K}$ was calculated from $(I-K)_{0}$ using the data in Houdashelt et al. (2000a, 2000b) and the bolometric luminosity was calculated from $K_{0}$ and $\mathrm{BC}_{K}$, with a distant modulus of the LMC 18.54 (Keller \& Wood 2006).

Finally, in Table 2, we indicate whether a star is below (a " 1 " in the last column) or above (a " 2 " in the last column) the higher-mass line in Figure 1. The last column in Table 2 also provides notes on unusual variability characteristics as given in Soszyński et al. (2004). Stars for which there is OGLE III data available are also indicated. The data in Table 2 are available online.

\subsection{Light Curves and Radial Velocity Curves}

We present samples of light and radial velocity curves for the ellipsoidal variables in Figure 2. Light and radial velocity curves for all objects are available as online data. The time series data for the light curves are from the MACHO, OGLE II, and OGLE III databases if they are available. Each radial velocity curve has at least 12 good quality data points in 1 orbital period, good enough for a binary orbital solution. As confirmed by Nicholls et al. (2010), our observed ellipsoidal variables show two light maxima and two minima in one orbital period, while the velocity shows only one maximum and one minimum. Among our 80 objects, most of them $(60 / 80=75 \%)$ have almost circular orbits, since they have equal light maxima and they have the same light curve widths for the first and second maxima (e.g., the top two objects of Figure 2). The other systems are eccentric binaries $(20 / 80=25 \%)$ since they show different light maxima 

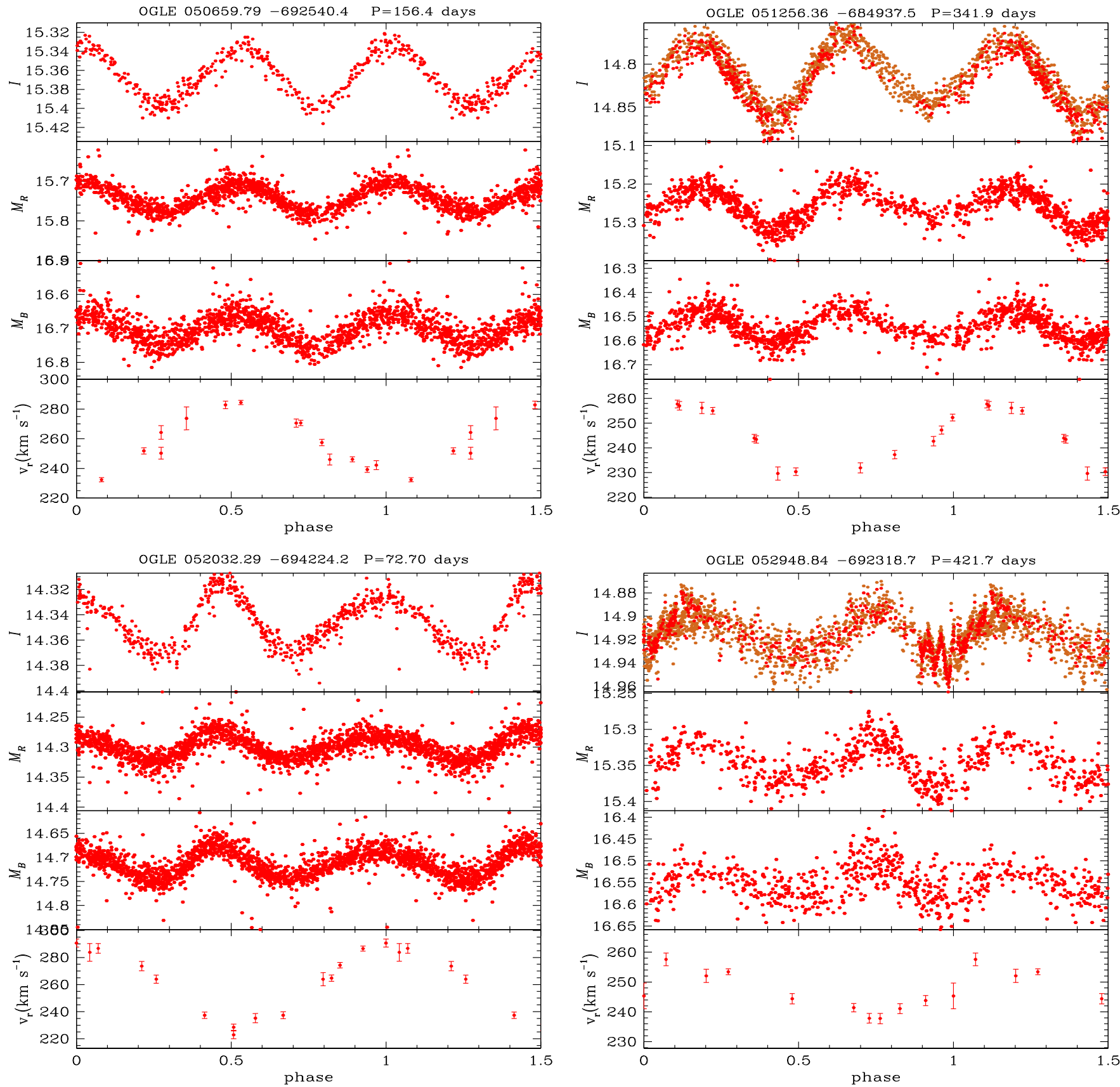

Figure 2. Light and radial velocity curves of ellipsoidal variables. $M_{R}$ and $M_{B}$ are the MACHO red and blue magnitudes, respectively. The top two objects are ellipsoidal variables with circular orbits while the bottom two have eccentric orbits. For the $I$ band data, light brown points are from OGLE III and red points are from OGLE II.

(A color version and the complete figure set (86 images) of this figure are available in the online journal.)

or light curve widths in one orbital period (e.g., the bottom two objects of Figure 2). In addition, there are binary systems that contain a semi-regular pulsating red giant $(8 / 80=10 \%)$ (e.g., object OGLE 052948.84 in Figure 2), similar to those noted by Nicholls \& Wood (2012). For these systems, both pulsation theory and binary theory can be used to constrain and compare stellar parameters.

\subsection{The Velocity Amplitude}

The distribution of full radial velocity amplitude $\Delta R V$ for the 80 ellipsoidal variables plus the combined sample from Nicholls et al. (2010) and Nicholls \& Wood (2012) is presented in the left panel of Figure 3 (red). From the figure, it can be seen that the velocity amplitude varies from $\sim 5$ to $\sim 130 \mathrm{~km} \mathrm{~s}^{-1}$, with a peak near $30 \mathrm{~km} \mathrm{~s}^{-1}$. Note that the number of stars with velocity amplitudes larger than $80 \mathrm{~km} \mathrm{~s}^{-1}$ is small.

Our observed velocity amplitude distribution is also compared to the model prediction of Nie et al. (2012) (the black dashed line in the left panel of Figure 3). The predicted distribution is for ellipsoidal red giant binaries on the top 1 magnitude of the RGB (870-2190 $\left.L_{\odot}\right)$ that have light amplitudes detectable by the OGLE II observations. Our comparison sub-sample of observations consists of 51 objects for which the luminosity lies on the top 1 magnitude of the RGB (the blue solid line in the left panel). Note that we compare to the model of Nie et al. 


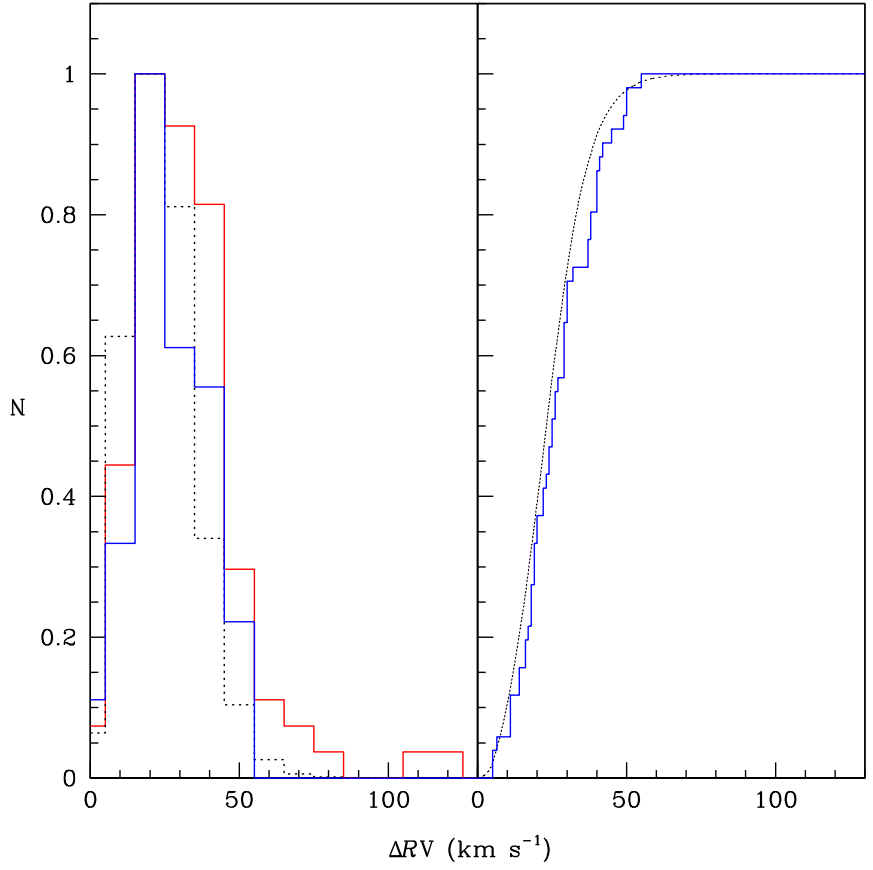

Figure 3. Distribution of the full radial velocity amplitude $\Delta R V$. Left panel the red solid line shows the distribution for the 80 ellipsoidal variables and the combined sample of Nicholls et al. (2010) and Nicholls \& Wood (2012), the blue solid line shows the observed distribution for 51 ellipsoidal variables on the top 1 magnitude of the RGB, and the black dashed line shows the predicted distribution for ellipsoidal variables on the top 1 magnitude of the RGB by the model of Nie et al. (2012) with the calibration of OGLE II data. Right panel: the predicted cumulative distribution for ellipsoidal variables on the top 1 magnitude of the RGB. Blue is for observations from 51 objects, and black is for the model of Nie et al. (2012) with the calibration of OGLE II data. Note that all the distribution peaks are normalized to unity.

(A color version of this figure is available in the online journal.)

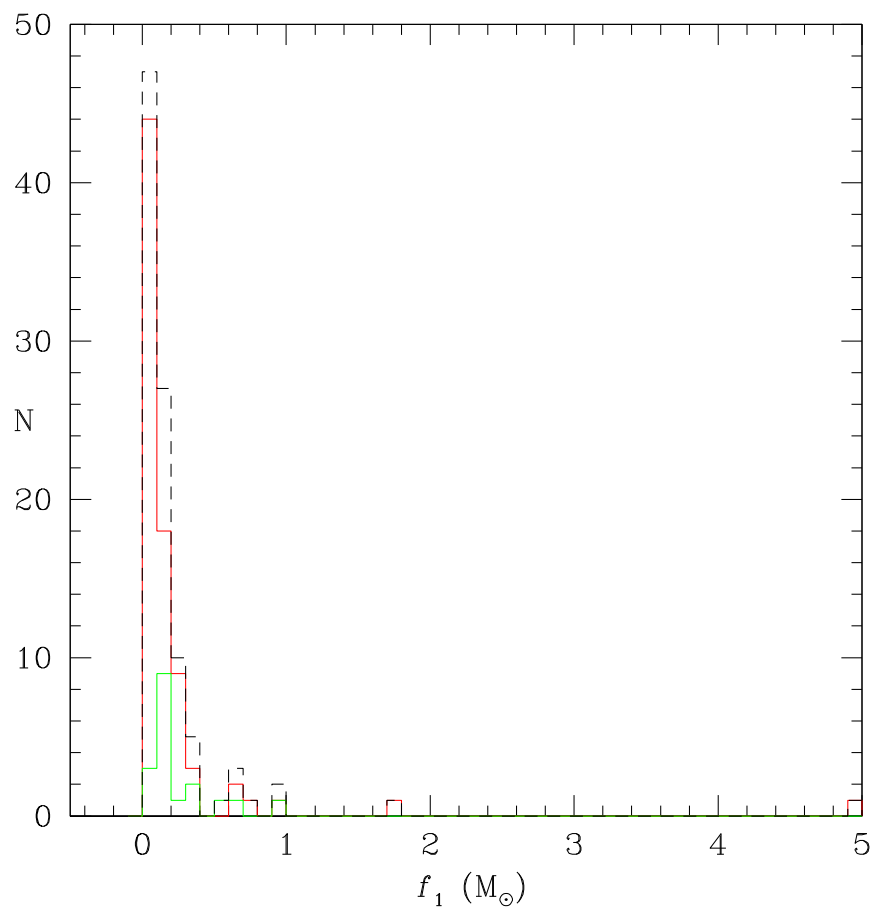

Figure 4. Binary mass function distribution for red giant ellipsoidal variables in the LMC. The red line denotes our observation, the green line denotes the combined observations of Nicholls et al. (2010) and Nicholls \& Wood (2012), and the black dashed line is the sum of the two distributions.

(A color version of this figure is available in the online journal.)

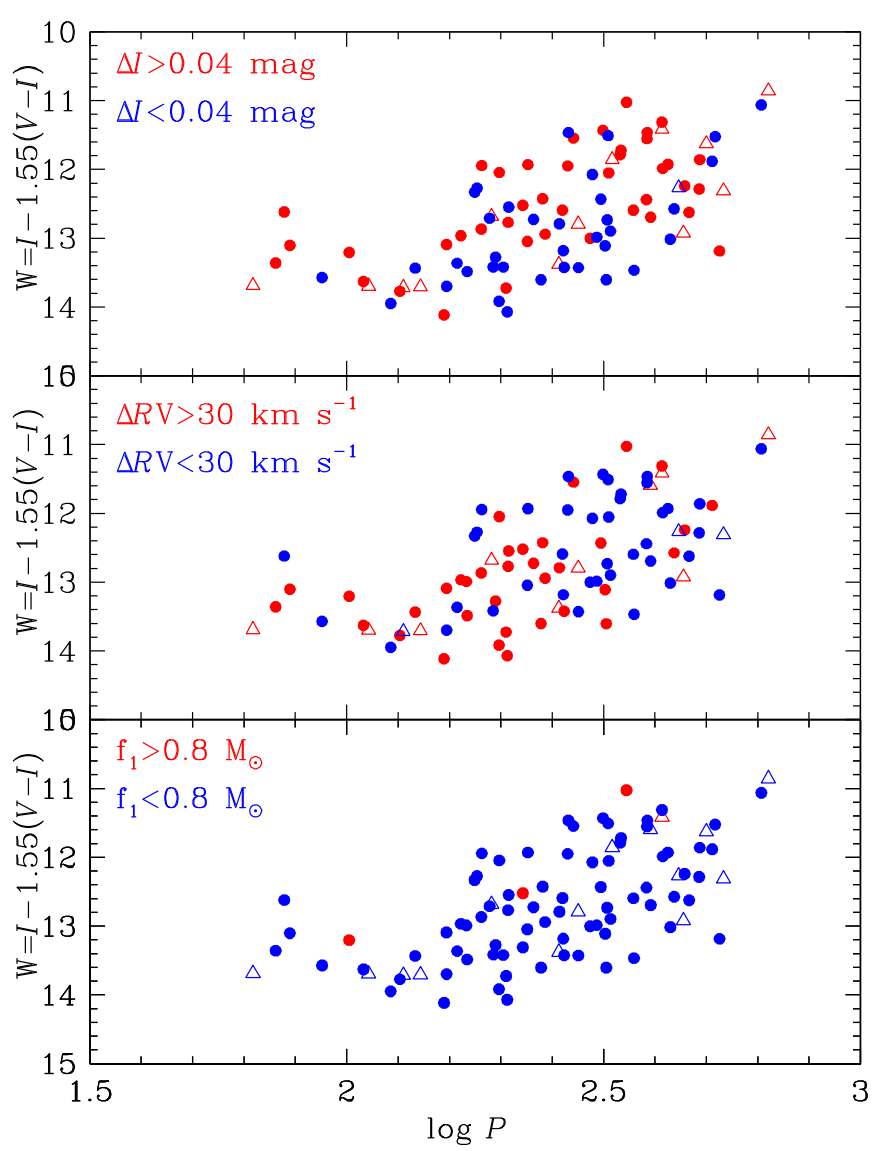

Figure 5. PL diagram for the our 80 ellipsoidal variables. The top panel shows the position on the PL diagram of objects with different full-amplitude $\Delta I$, the middle panel shows the position of objects with different full-amplitude $\Delta R V$, and the bottom panel shows the position of objects with different mass function $f_{1}$. In all panels, filled circles denote observations from this work and open triangles denote observations from Nicholls et al. (2010) and Nicholls \& Wood (2012).

(A color version of this figure is available in the online journal.)

(2012), which is calibrated on the OGLE II data of Soszyński et al. (2004) because that calibration is better than the one based on MACHO data due to the extra sensitivity of OGLE II observations to small-amplitude light variations.

The cumulative distributions corresponding to the histograms of the ellipsoidal variables on the top 1 magnitude of the RGB in Figure 3 are shown in the right panel. A two-sample Kolmogorov-Smirnov test gives a probability of up to 0.20 that the observation and model come from the same underlying distribution. There is thus a modest probability that the model is consistent with both the OGLE II photometry and the velocity amplitudes derived in this study.

\subsection{The Mass Function}

The binary mass function $f_{1}$ of a binary system where the primary star (in our case, the red giant) is observable is

$$
f_{1}=\frac{K_{1}^{3} P}{2 \pi G}=\frac{m_{2}^{3} \sin ^{3} i}{\left(m_{1}+m_{2}\right)^{2}},
$$

where $K_{1}$ is the radial velocity semi-amplitude of the primary star, $P$ is the orbital period, $m_{1}$ is the mass of the primary star, and $m_{2}$ is the mass of the secondary star. Note that $f_{1}$ is a minimum estimate for $m_{2}$. The distribution of $f_{1}$ for our sample of red giant ellipsoidal variables in the LMC is shown in Figure 4. The 
Table 3

Properties of Objects Rejected

\begin{tabular}{|c|c|c|c|c|c|c|c|c|c|c|c|c|c|}
\hline No. & $\begin{array}{c}\text { Object } \\
\text { (OGLE II Name) }\end{array}$ & $\begin{array}{c}P \\
\text { (day) }\end{array}$ & $\begin{array}{c}I \\
(\mathrm{mag})\end{array}$ & $\begin{array}{c}V \\
(\mathrm{mag})\end{array}$ & $\begin{array}{c}M_{B} \\
(\mathrm{mag})\end{array}$ & $\begin{array}{c}M_{R} \\
(\mathrm{mag})\end{array}$ & $\begin{array}{c}K \\
(\mathrm{mag})\end{array}$ & $\begin{array}{c}\Delta I \\
(\mathrm{mag})\end{array}$ & $\begin{array}{c}\Delta R V \\
\left(\mathrm{~km} \mathrm{~s}^{-1}\right)\end{array}$ & $\begin{array}{c}L \\
\left(L_{\odot}\right)\end{array}$ & $\begin{array}{l}T_{\text {eff }} \\
(\mathrm{K})\end{array}$ & $\begin{array}{c}R \\
\left(R_{\odot}\right)\end{array}$ & Remark \\
\hline 1 & $050716.07-693259.7$ & 563.3 & 14.81 & 16.18 & $\cdots$ & $\cdots$ & 13.09 & 0.025 & $\cdots$ & 1571 & 4368 & 70 & 1 \\
\hline 2 & 051111.34-693714.9 & 147.7 & 15.69 & 17.41 & $\cdots$ & $\cdots$ & 13.59 & 0.060 & $\cdots$ & 772 & 3984 & 59 & 1 \\
\hline 3 & $051150.57-685627.0$ & 119.3 & 15.55 & 16.91 & 16.73 & 15.77 & 13.80 & 0.025 & $\cdots$ & 804 & 4344 & 51 & 1 \\
\hline 4 & $052346.66-695140.6$ & 259.0 & 15.34 & 16.77 & $\ldots$ & $\cdots$ & 13.54 & 0.050 & $\cdots$ & 983 & 4290 & 57 & $1,+$ sr,ogle 3 \\
\hline 5 & $053500.22-702643.4$ & 286.5 & 15.19 & 16.71 & 16.60 & 15.52 & 13.34 & 0.060 & $\cdots$ & 1141 & 4225 & 64 & 1, ogle3 \\
\hline 6 & $054109.54-704610.5$ & 378.8 & 14.81 & 17.17 & 17.30 & 15.55 & 12.05 & 0.200 & $\cdots$ & 2298 & 3517 & 130 & 1,ogle 3 \\
\hline
\end{tabular}

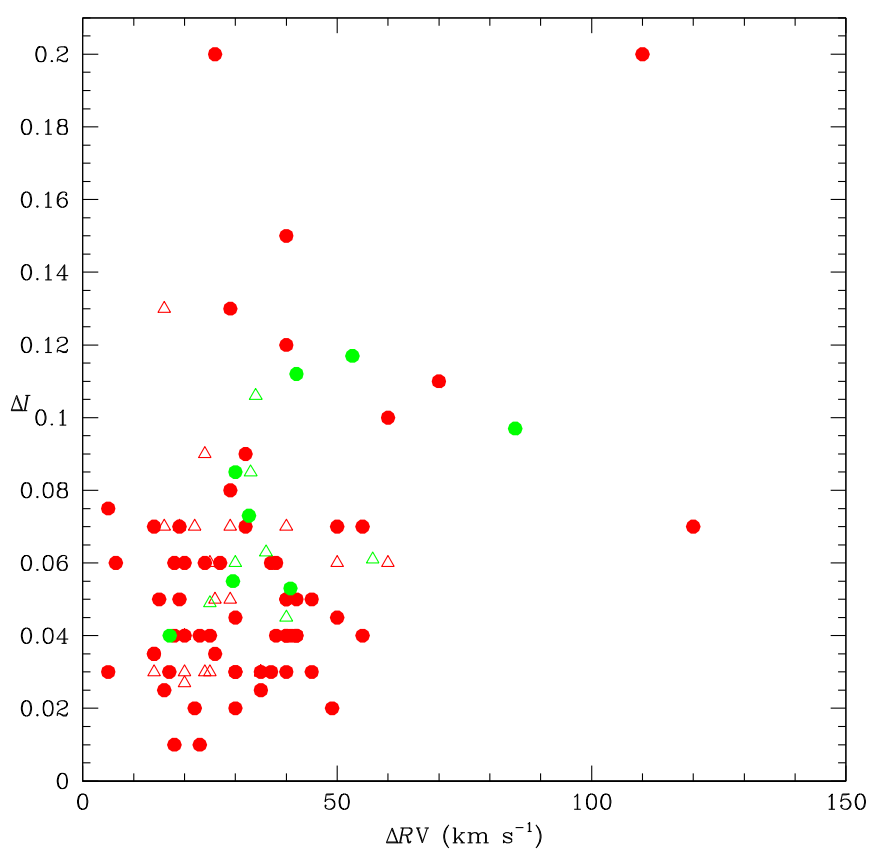

Figure 6. Relation between the velocity amplitude and light amplitude. Red: observations from this work; circles are for circular binaries and triangles are for eccentric binaries. Green: observations from Nicholls et al. (2010) and Nicholls $\&$ Wood (2012); circles are for circular binaries and triangles are for eccentric binaries. Note that some objects are overwritten because they have the same velocity and light curve amplitude.

(A color version of this figure is available in the online journal.)

figure shows a peak at about $0.2 M_{\odot}$, presumably due to the dominant low-mass population, but there are a few objects that must be in more massive binaries where the secondary star is of intermediate mass with $m_{2} \gtrsim 4 M_{\odot}$. Red giants of this mass were found in ellipsoidal binaries in the LMC by Nicholls \& Wood (2012). If we assume a random pole orientation for the binary orbit, which implies that the mean of $\sin ^{3} i=3 \pi / 16$, and if we also assume that the mean mass ratio is $q=1$, then for stars in the peak of the distribution around $f_{1} \sim 0.2 M_{\odot}$, the mean mass of the red giant is $m_{1} \sim 1.35 M_{\odot}$. This is similar to peak in the mass distribution of red giants predicted by modeling the star formation history of the LMC (e.g., Nie et al. 2012).

\subsection{The PL Diagram}

The variations of light amplitude, velocity amplitude, and mass function across the PL diagram are shown in Figure 5. In the top panel, we can see that the higher-amplitude ellipsoidal variables tend to lie on the higher luminosity, shorter period side of sequence E. This is just as demonstrated by Soszyński et al. (2004) and is presumably because as the red giant in a binary system (with a given orbital period) evolves to higher

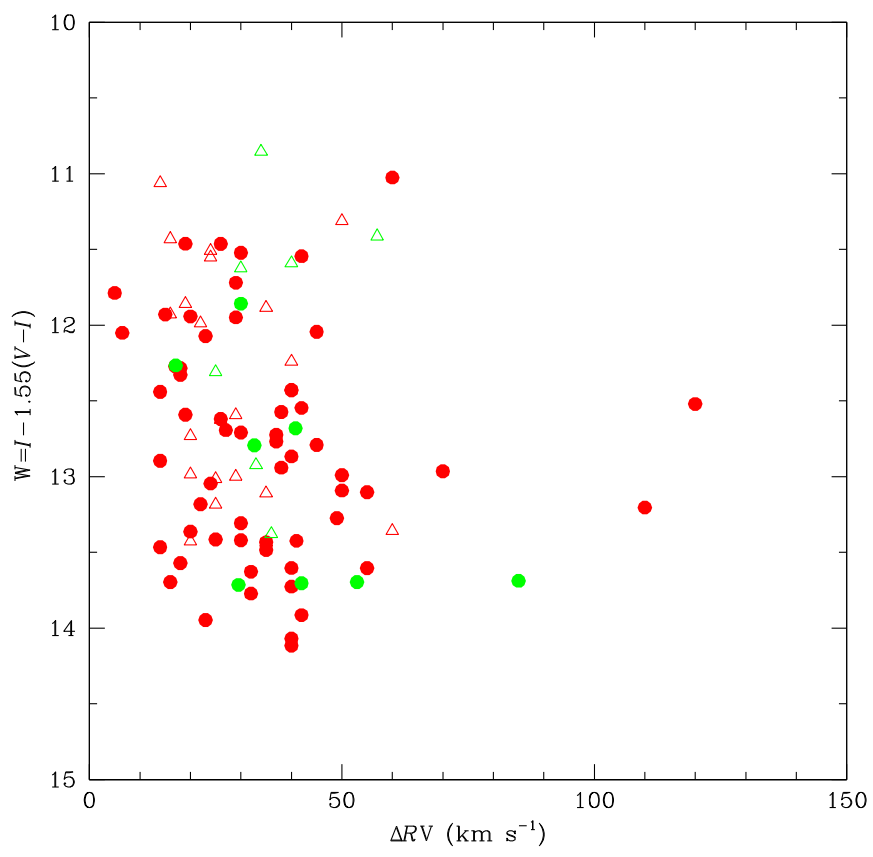

Figure 7. Relation between velocity amplitude and luminosity. Symbols are as in Figure 6.

(A color version of this figure is available in the online journal.)

luminosity, it will expand to a greater filling fraction of its Roche lobe and hence be more distorted and have a large light variation amplitude.

The middle panel of Figure 5 shows a tendency for highervelocity amplitudes to be associated with shorter-period orbits, as might be expected. Of course, low-velocity amplitudes can be seen for shorter-period orbits if the orbital plane is oriented close to the plane of the sky: there are a few objects that seem to fall in this category.

The mass function also shows some correlation with position in the PL diagram (bottom panel of Figure 5). The most massive objects, with $f_{1}>0.8 M_{\odot}$, lie on the upper part of sequence $\mathrm{E}$ in agreement with the results shown in Figure 1.

\subsection{Velocity Amplitude versus Light Amplitude}

In Figure 6, we present the light amplitude as a function of the velocity amplitude. Red symbols are our observation, and green symbols are observations from Nicholls et al. (2010) and Nicholls \& Wood (2012). The 20 eccentric ellipsoidal variables from our observations and the 7 highly eccentric ellipsoidal variables studied by Nicholls \& Wood (2012) are marked as open triangles. As expected, there is generally no correlation of the light and velocity amplitudes. The former depends on the Roche lobe filling factor (and inclination), while the latter 

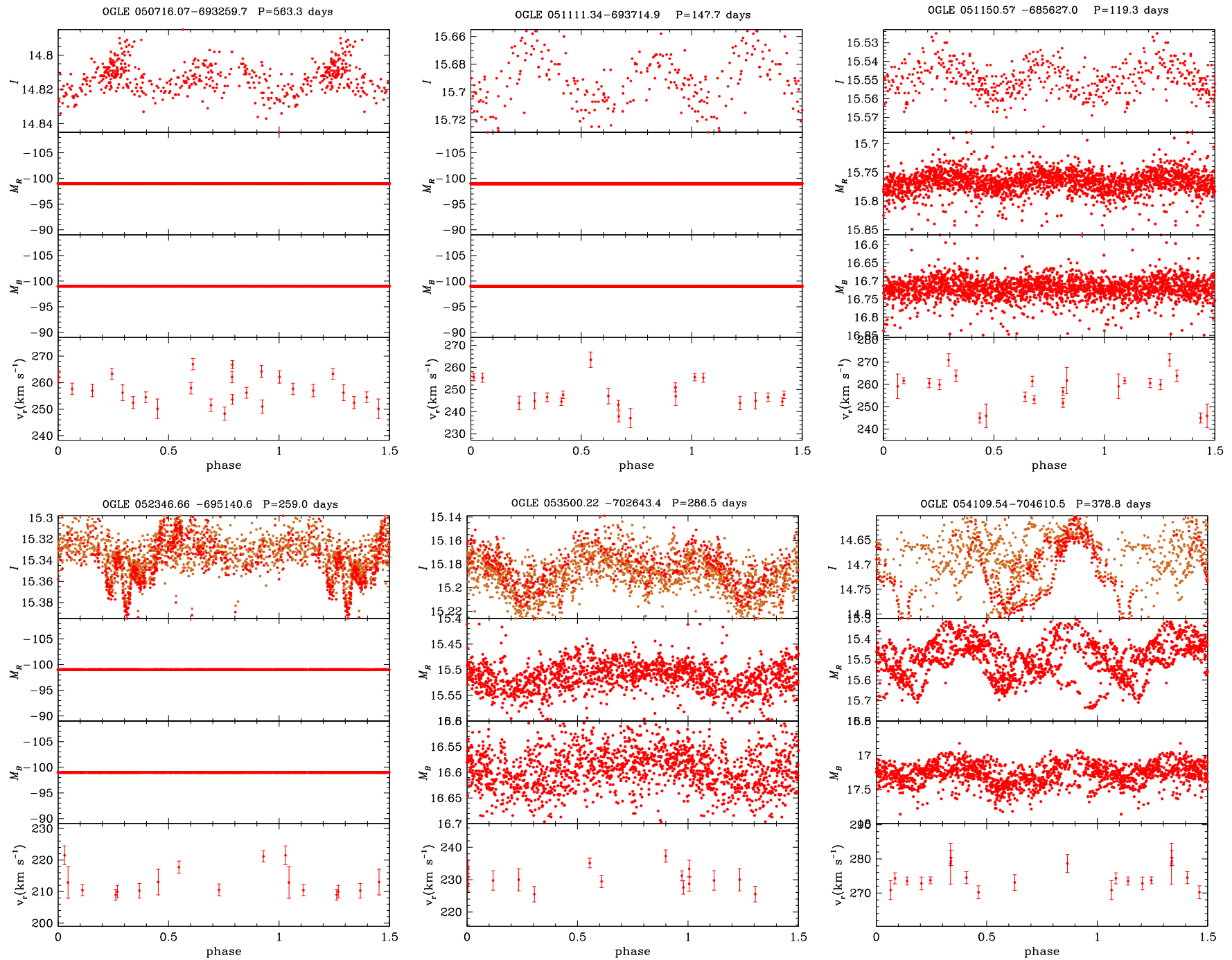

Figure 8. Light and radial velocity curves of the six objects in the Appendix.

(A color version of this figure is available in the online journal.)

depends on the stellar masses and the orbital separation. In principal, the orbital separation could influence the Roche lobe filling factor, but in practice the orbital separation effectively determines the luminosity on the giant branch where the Roche lobe nearly fills and ellipsoidal variability becomes detectable. This suggests that the orbital separation (velocity amplitude) should depend on luminosity for red giant ellipsoidal variables, but the light amplitude should not depend on orbital separation (velocity amplitude).

\subsection{Velocity Amplitude versus Luminosity}

As suggested in the last section, the velocity amplitude should show some correlation with luminosity. In Figure 7, we show the dependence of luminosity on velocity amplitude. The binaries with larger velocity amplitudes do tend to have lower luminosities, although the effect is not prominent. This is as expected, since higher-velocity amplitude generally means a smaller separation and thus Roche lobe filling should occur at a lower luminosity.

\section{SUMMARY AND CONCLUSIONS}

We have presented radial velocity observations obtained for 80 ellipsoidal red giant binaries in the LMC. This sample is much larger than the previous samples of Nicholls et al. (2010) and Nicholls \& Wood (2012). The mass function of the sample suggests that the typical mass of the red giants in the ellipsoidal binaries is $\sim 1.35 M_{\odot}$, in agreement with estimates derived from the star formation history of the LMC.

The main purpose of this paper was to present radial velocity data and basic observational properties of 80 ellipsoidal variables in the LMC. In future work, the radial velocity data will be combined with MACHO and OGLE photometric light curve data to give complete orbital solutions for these binary systems. This is possible because the distance, and hence the luminosity, of these LMC objects is well known (e.g., Nicholls $\&$ Wood 2012). The results of the complete solutions will yield statistical distributions of masses, mass ratios, separations, and eccentricities in the period range observed. These distributions of orbital elements for binaries in the LMC can be compared with the solar vicinity statistical data given in the classic paper of Duquennoy \& Mayor (1991) and the recent paper by Raghavan et al. (2010). It will be interesting to see if the same distributions exists in samples of binaries with different metallicity distributions and different star formation histories.

We acknowledge constructive comments by the anonymous referee. J.D.N. thanks Geoff White, Donna Burton, Catherine 
Farage, and the other technical staff at Siding Springs Observatory (SSO) for their assistance and support throughout this observing project. J.D.N. is supported by the National Natural Science Foundation of China (NSFC) through grant 11303043 and the Young Researcher Grant of National Astronomical Observatories, Chinese Academy of Sciences. P.R.W. received partial support for this project from Australian Research Council Discovery Project grant DP120103337.

\section{APPENDIX LIST OF OBJECTS REJECTED}

Here we present data for the six objects that we removed from the 86 ellipsoidal candidates due to their poor velocity data relative to the noise. Properties of these six stars are given in Table 3, and their light and velocity curves are presented in Figure 8 . Their velocity data is given in Table 1.

\section{REFERENCES}

Adams, E., Wood, P. R., \& Cioni, M.-R. 2006, MmSAI, 77, 537

Allen, D. A. 1984, PASAu, 5, 369

Belczyński, K., Mikołajewska, J., Munari, U., Ivison, R. J., \& Friedjung, M. 2000, A\&AS, 146, 407

Cutri, R. M., Skrutskie, M. F., van Dyk, S., et al. 2003, yCat, 2246, 0
Dopita, M., Hart, J., McGregor, P., et al. 2007, Ap\&SS, 310, 255

Dopita, M., Rhee, J., Farage, C., et al. 2010, Ap\&SS, 327, 245

Duquennoy, A., \& Mayor, M. 1991, A\&A, 248, 485

Fraser, O. J., Hawley, S. L., \& Cook, K. H. 2008, AJ, 136, 1242

Houdashelt, M. L., Bell, R. A., \& Sweigart, A. V. 2000b, AJ, 119, 1448

Houdashelt, M. L., Bell, R. A., Sweigart, A. V., \& Wing, R. F. 2000a, AJ, 119,1424

Ita, Y., Tanabé, T., Matsunaga, N., et al. 2004, MNRAS, 353, 705

Keller, S. C., \& Wood, P. R. 2006, ApJ, 642, 834

Kenyon, S. J. 1986, The Symbiotic Stars (Cambridge: Cambridge Univ. Press)

Madore, B. F. 1982, ApJ, 253, 575

Mikolajewska, J., Acker, A., \& Stenholm, B. 1997, A\&A, 327, 191

Nicholls, C. P., \& Wood, P. R. 2012, MNRAS, 421, 2616

Nicholls, C. P., Wood, P. R., \& Cioni, M.-R. L. 2010, MNRAS, 405, 1770

Nicholls, C. P., Wood, P. R., Cioni, M.-R. L., \& Soszyński, I. 2009, MNRAS, 399, 2063

Nie, J. D., Wood, P. R., \& Nicholls, C. P. 2012, MNRAS, 423, 2764

Raghavan, D., McAlister, H. A., Henry, T. J., et al. 2010, ApJS, 190, 1

Rieke, G. H., \& Lebofsky, M. J. 1985, ApJ, 288, 618

Schlegel, D. J., Finkbeiner, D. P., \& Davis, M. 1998, ApJ, 500, 525

Soszynski, I., Dziembowski, W. A., Udalski, A., et al. 2007, AcA, 57, 201

Soszyński, I., Udalski, A., Kubiak, M., et al. 2004, AcA, 54, 347

Stellingwerf, R. F. 1978, ApJ, 224, 953

Wilson, R. E. 1979, ApJ, 234, 1054

Wilson, R. E. 1990, ApJ, 356, 613

Wilson, R. E., Chochol, D., Komžík, R., et al. 2009, ApJ, 702, 403

Wilson, R. E., \& Devinney, E. J. 1971, ApJ, 166, 605

Wood, P. R., Alcok, C., Allsman, R. A., et al. 1999, in IAU Symp. 191, Asymptotic Giant Branch Stars, ed. T. Le Bertre, A. Lèbre, \& C. Waelkens (Cambridge: Cambridge Univ. Press), 151 